\title{
Changes in pulmonary exercise haemodynamics in scleroderma: a 4-year prospective study
}

\author{
Gabor Kovacs ${ }^{1,2}$, Alexander Avian ${ }^{3}$, Nora Wutte ${ }^{4}$, Franz Hafner ${ }^{5}$, \\ Florentine Moazedi-Fürst ${ }^{6}$, Sonja Kielhauser ${ }^{6}$, Elisabeth Aberer ${ }^{4}$, \\ Marianne Brodmann ${ }^{5}$, Winfried Graninger ${ }^{6}$, Vasile Foris ${ }^{1}$, \\ Andrea Olschewski ${ }^{2,7}$ and Horst Olschewski ${ }^{1,2}$
}

\begin{abstract}
Affiliations: 'Dept of Internal Medicine, Division of Pulmonology, Medical University of Graz, Graz, Austria. ${ }^{2}$ Ludwig Boltzmann Institute for Lung Vascular Research, Graz, Austria. ${ }^{3}$ Institute for Medical Informatics, Statistics and Documentation, Medical University of Graz, Graz, Austria. ${ }^{4}$ University Clinic for Dermatology, Medical University of Graz, Graz, Austria. ${ }^{5}$ Dept of Internal Medicine, Division of Angiology, Medical University of Graz, Graz, Austria. 'Dept of Internal Medicine, Division of Rheumatology, Medical University of Graz, Graz, Austria. ${ }^{7}$ Institute of Physiology, Medical University of Graz, Graz, Austria.
\end{abstract}

Correspondence: Gabor Kovacs, Ludwig Boltzmann Institute for Lung Vascular Research, Stiftingtalstrasse 24, 8010 Graz, Austria. E-mail: gabor.kovacsQklinikum-graz.at

@ERSpublications

Scleroderma patients without pulmonary hypertension develop mild deterioration in pulmonary exercise haemodynamics http://ow.ly/hva930aEW4E

Cite this article as: Kovacs G, Avian A, Wutte N, et al. Changes in pulmonary exercise haemodynamics in scleroderma: a 4-year prospective study. Eur Respir J 2017; 50: 1601708 [https://doi.org/10.1183/ 13993003.01708-2016].

ABSTRACT Pulmonary arterial hypertension (PAH) is a feared complication of systemic sclerosis. In this prospective cohort study, we monitored the changes in resting and exercise pulmonary haemodynamics of scleroderma patients without initial PAH over a mean follow-up period of $\sim 4$ years.

All patients underwent exercise echocardiography and cardiopulmonary exercise testing at baseline and follow-up. A subgroup underwent exercise right heart catheter (RHC) investigations. The primary endpoint was the echocardiographic systolic pulmonary arterial pressure at $50 \mathrm{~W}$ exercise (sPAP50).

We included 99 patients, of whom 58 had a complete dataset. Three out of 99 patients developed RHCconfirmed PAH $(0.75$ cases per 100 patient-years). sPAP50 increased $(\mathrm{p}<0.001)$ and peak oxygen uptake (secondary end-point) decreased significantly $(\mathrm{p}=0.001)$ during follow-up, but there was no significant change in resting sPAP ( $\mathrm{p}=0.38)$. In the RHC subgroup $(\mathrm{n}=28)$, mean $(\mathrm{m})$ PAP and pulmonary vascular resistance at $50 \mathrm{~W}$ increased significantly $(\mathrm{p}=0.02$ and $\mathrm{p}=0.002$, respectively), but resting mPAP was unchanged.

Scleroderma patients without PAH develop a mild but significant deterioration of pulmonary exercise haemodynamics and exercise capacity over a 4-year follow-up period, indicating a progression of pulmonary vascular disease. The manifestation rate of $\mathrm{RHC}$-confirmed $\mathrm{PAH}$ was 0.75 cases per 100 patient-years.

\footnotetext{
This article has supplementary material available from erj.ersjournals.com

Received: Aug 282016 | Accepted after revision: April 022017

This study is registered at clinicaltrials.gov with identifier NCT01468792.

Conflict of interest: Disclosures can be found alongside this article at erj.ersjournals.com

Copyright OERS 2017
} 


\section{Introduction}

Pulmonary arterial hypertension (PAH) is a feared complication of systemic sclerosis. The prevalence of haemodynamically proven precapillary pulmonary hypertension in large cohorts of scleroderma patients ranges from $5 \%$ to $12 \%$ [1-5], while the estimated incidence of $\mathrm{PAH}$ among systemic sclerosis patients is between 0.61 and two cases per 100 patient-years [6-10]. PAH is considered to be the primary cause of death in $14 \%$ of scleroderma patients [11], indicating significantly poorer survival than in scleroderma patients without PAH [3, 11]. Moreover, systemic sclerosis-associated PAH has been shown to have an even worse prognosis than idiopathic PAH [12-14]. Screening programmes have been implemented in order to recognise systemic sclerosis-associated PAH at an early stage, in the hope that this may lead to better therapy responses and improved survival $[4,15,16]$.

Clinical signs and markers including decreased diffusion capacity of the lung for carbon monoxide (DLCO) [9, 17, 18], forced vital capacity [9], exercise hypoxia [18], the presence of anti-RNA polymerase or anti-U3RNP antibodies [7] and increased N-terminal pro-brain natriuretic peptide (NT-proBNP) [17] may predict the development of $\mathrm{PAH}$ in systemic sclerosis. In addition, a strong increase in pulmonary arterial pressure (PAP) during exercise, which may indicate stiffening of the pulmonary arteries, was shown to be an independent predictor of PAH $[8,19]$ and was associated with decreased exercise capacity and poor outcome $[20,21]$. Altogether, changes in pulmonary exercise haemodynamics over time may reveal important information on the development of pulmonary vascular disease in scleroderma patients. In this study, we investigated the changes in pulmonary haemodynamics by means of echocardiography and right heart catheterisation (RHC) at rest and during exercise in patients with systemic sclerosis. To our knowledge, this is the first follow-up study of exercise pulmonary haemodynamics in such a patient population. We hypothesised that exercise PAP values increase over time, indicating the progression of pulmonary vascular disease.

\section{Patients and methods}

Our study included patients with systemic sclerosis who took part in our previous screening programmes $[20,22]$ and underwent exercise echocardiography. The time between the baseline and follow-up investigation was $\geqslant 3$ years. Patients with known PAH or those with severe cardiac (systolic or diastolic left ventricular failure (ejection fraction $<50 \%$ or diastolic failure $>$ mild), significant valvular disease or systemic arterial hypertension (resting systolic values $>150 \mathrm{mmHg}$ or resting diastolic values $>90 \mathrm{mmHg}$ on medication)) or pulmonary comorbidities (forced expiratory volume in $1 \mathrm{~s}<60 \%$ predicted) at baseline were excluded. Written informed consent was obtained from all patients and the study was conducted in line with the Helsinki declaration.

All patients underwent routine laboratory and pulmonary function tests including the determination of DLCO as well as resting echocardiography. All patients underwent exercise echocardiography during a symptom-limited cardiopulmonary exercise test on a variable-load, half-recumbent, left-sloping echocardiography cycle ergometer (ER 900 EL; Ergoline; Bitz, Germany) using a standard protocol with a $25 \mathrm{~W}$ workload increment every $2 \mathrm{~min}$. Doppler signals were continuously recorded. Recordings at rest and during exercise were analysed off-line in random order and in a blinded fashion. Tricuspid regurgitation was visualised from the apical four-chamber view. Systolic pulmonary arterial pressure (sPAP) was estimated from the peak tricuspid regurgitation jet, using the simplified Bernoulli equation $\left(\mathrm{sPAP}=4 \times \mathrm{v}^{2}+\right.$ right atrial pressure), where " $\mathrm{v}$ " is the peak velocity of the tricuspid regurgitation jet $\left(\mathrm{m} \cdot \mathrm{s}^{-1}\right)$, and the right atrial pressure is estimated from the diameter and breath-induced variability of the inferior vena cava.

In patients with suspected pulmonary hypertension, based on resting echocardiography [1] or a strong increase of PAP during exercise $(>50 \mathrm{mmHg}$ ), or unexplained exercise limitation during cardiopulmonary exercise testing, RHC at rest and during exercise was suggested. RHC examinations were performed using a 7 F, quadruple lumen, balloon tipped, flow-directed Swan-Ganz catheter (Edwards Lifesciences, Irvine, CA, USA) using the transjugular approach. The reference point was set at mid-thoracic level, as suggested previously [23]. Pressures were continuously recorded and averaged over several respiratory cycles during spontaneous breathing, during both rest and exercise. Parameters obtained at rest included systolic, diastolic and mean (m)PAP, pulmonary arterial wedge pressure (PAWP), right atrial pressure and cardiac output measured using the thermodilution technique. Pulmonary vascular resistance (PVR) was derived from the difference between mPAP and PAWP divided by cardiac output. Oxygen tension $\left(P_{\mathrm{O}_{2}}\right)$ and oxygen saturation of arterialised ear lobe capillary blood and pulmonary arterial blood were determined using an ABL 800 Flex (Radiometer, Copenhagen, Denmark) blood gas analyser. Systemic blood pressure was measured using a sphygmomanometer. After baseline measurements, patients underwent a symptom-limited exercise test on the same cycle ergometer and using the same exercise protocol as during exercise echocardiography. Systolic, diastolic and mean PAP and corresponding systemic blood pressure 
values, as well as PAWP and right atrial pressure, cardiac output, capillary and central venous $\mathrm{PO}_{2}$ and oxygen saturation were measured at each stage of exercise and at 3 and $8 \mathrm{~min}$ after exercise. All examinations and measurements were performed by the same experienced team. There were no complications.

The predefined primary end-point of our study was the change in sPAP at $50 \mathrm{~W}$ (sPAP50) over the follow-up period. The secondary end-point was the change in peak oxygen uptake between the baseline and follow-up examination, as a measure of exercise capacity. In the subgroup of patients with at least two RHC examinations, in addition to exercise echocardiography, the change in mPAP at rest, at $50 \mathrm{~W}$ and PVR at $50 \mathrm{~W}$ from baseline to follow-up represented further end-points.

Data are presented as mean $\pm \mathrm{SD}$ or mean $(95 \% \mathrm{CI})$ or median and interquartile range for continuous variables and absolute and relative frequency for categorical data. Patients' characteristics were compared using t-tests, the Mann-Whitney U-test, Chi-squared test and Fisher's exact test. Changes were analysed using the Wilcoxon signed-rank test or paired t-test, as appropriate. A p-value $<0.05$ was considered significant. Statistical analysis was performed using SPSS Statistics (release 22.0.0. 2013; IBM, Chicago, IL, USA) software.

\section{Results}

We enrolled 99 patients, out of whom 58 (limited cutaneous systemic sclerosis $76 \%$, diffuse cutaneous systemic sclerosis $16 \%$ and mixed connective tissue disease $9 \%$ ) underwent follow-up examination. All of the 58 patients had a complete dataset (figure 1; patients' characteristics are shown in table 1 and online supplementary tables S1 and S2). The mean time interval between the two examinations was $3.9 \pm 0.9$ years. During this period, three out of 99 patients developed RHC-confirmed PAH (0.75 cases per 100 patient-years). There was a significant increase in sPAP50 and a significant decrease in peak oxygen uptake between the two examinations (primary and secondary end-points, $p<0.001$ and $p=0.001$, respectively; table 2 and figure 2). There was no significant change in resting $\operatorname{sPAP}(\mathrm{p}=0.38 ;$ fig. 2). NT-proBNP showed a small statistically significant increase during follow-up, while patients' pulmonary function tests showed no significant changes (table 2). There was no relevant correlation between baseline characteristics and changes in sPAP or peak oxygen uptake during follow-up (online supplementary table S3). Out of the 58 patients, 28 underwent at least two RHC examinations (mean interval between examinations $2.4 \pm 1.3$ years). Within this subgroup, there was a significant increase in mPAP at $50 \mathrm{~W}$ $(\mathrm{p}=0.02), P V R$ at $50 \mathrm{~W}(\mathrm{p}=0.002)$ and in the $\mathrm{mPAP} / \mathrm{cardiac}$ output slope $(\mathrm{p}=0.049)$, while there was no significant change in resting $\mathrm{mPAP}, \mathrm{PAWP}$ or PAWP/cardiac output (table 3 and figure 3 ). At baseline RHC, eight out of 28 patients, and at follow-up catheterisation, 11 out of 28 patients revealed a mPAP $>30 \mathrm{mmHg}$ and a total pulmonary resistance $>3$ Woods units during maximal exercise. There was no significant difference between these patients and those not fulfilling these criteria regarding their change in resting or exercise haemodynamics during follow-up (online supplementary table S4).

\section{Discussion}

In this study, the changes in pulmonary exercise haemodynamics were assessed by exercise echocardiography and RHC in patients with systemic sclerosis $\sim 4$ years after their baseline examination. To our knowledge, this is the first long-term study with follow-up exercise pulmonary haemodynamic data from scleroderma patients. We found a significant increase in PAP, PVR, and the PAP/cardiac output slope during exercise and a significant decrease in exercise capacity, indicating a progression towards

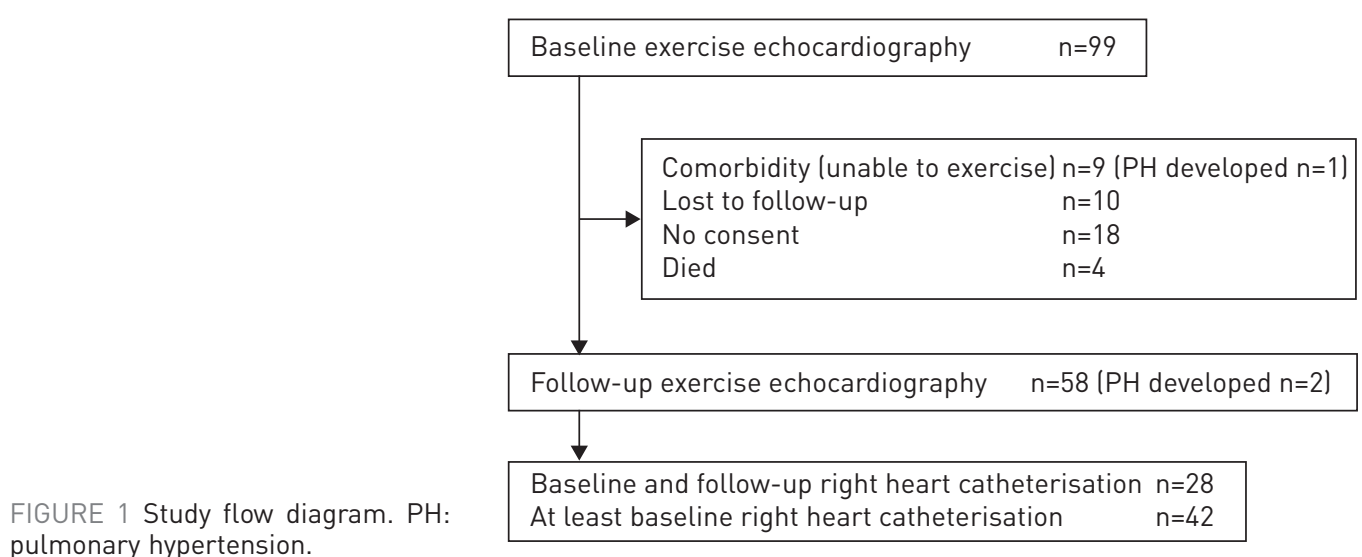


TABLE 1 Characteristics of patients with baseline and follow-up exercise echocardiography

\begin{tabular}{lc} 
Subjects $\mathbf{n}$ & 58 \\
Height $\mathbf{c m}$ & $166.2 \pm 7.0$ \\
Weight kg & $67.7 \pm 13.6$ \\
Sex & $6(10.3)$ \\
$\quad$ Male & $52(89.7)$ \\
Female & $3.9 \pm 0.9$ \\
Time between exercise echo studies years & $2.4 \pm 1.3$ \\
Time between RHC studies years & $46.7 \pm 11.3$ \\
Age at diagnosis years & $46.9 \pm 11.7$ \\
Age at first non-Raynaud symptom years & \\
Age years & $51.3 \pm 11.5$ \\
$\quad$ Baseline & $55.3 \pm 11.6$ \\
Follow-up & \\
Time since diagnosis years & $3.0(1.1-6.9)$ \\
$\quad$ Baseline & $6.7(5.2-11.5)$ \\
Follow-up & \\
Patients on bosentan therapy & $4(6.9)$ \\
$\quad$ Baseline & $16(27.6)$ \\
Follow-up & \\
Patients on immunosuppressive therapy & $17(29.3)$ \\
$\quad$ Baseline & $18(31.0)$ \\
Follow-up & \\
Uric acid mg-dL & \\
$\quad$ Baseline & $4.5(3.9-5.2)$ \\
Follow-up & $4.7(4.0-5.6)$ \\
Patients with ACA positivity & \\
$\quad$ Baseline & $30(51.7)$ \\
Follow-up & $33(56.9)$ \\
Patients with telangiectasis & \\
$\quad$ Baseline & $26(44.8)$ \\
Follow-up & $29(50.0)$ \\
Rodnan skin thickness score & \\
$\quad$ Baseline & $7.3 \pm 8.1$ \\
Follow-up & $6.7 \pm 6.7$ \\
\hline
\end{tabular}

Data are presented as mean \pm SD, $\mathrm{n}(\%)$ or median (interquartile range), unless otherwise stated. RHC: right heart catheterisation; ACA: anticentromere antibody.

pulmonary vascular disease. RHC-confirmed PAH developed in three out of 99 patients, corresponding to $\geqslant 0.75$ cases per 100 patient-years, which is in line with previous reports [6-10].

\section{Exercise haemodynamics in systemic sclerosis}

In recent years, growing evidence has accumulated that pulmonary exercise haemodynamics [24, 25] may be important for recognising early pulmonary vascular disease in systemic sclerosis $[8,19,20]$. RHC represents the most reliable method to assess pulmonary haemodynamics during exercise, while exercise echocardiography has been considered for screening purposes where it may provide valuable data in experienced hands [22]. In several studies, a strong increase in PAP during exercise was described in a large proportion of scleroderma patients [26-30], but there are few data regarding the change in these parameters over the years. In this study, we prospectively performed baseline and follow-up exercise echocardiography in all enrolled patients and assessed the changes in pulmonary haemodynamics during exercise; almost half of the subjects were additionally examined using invasive measurements confirming all the major findings of the noninvasive assessment. At RHC, besides the increase in absolute PAP values during exercise, we observed a mild but significant increase in the PAP/cardiac output slope between the two examinations. Generally, these slopes were much steeper than those derived from healthy control subjects $[24,31]$.

Several underlying mechanisms may cause a strong increase in PAP during exercise. In systemic sclerosis patients, the causes of exercise-induced pulmonary hypertension may be quite heterogeneous, with latent left heart disease being the most common single cause [32-36]. This might be supported by the increasing number of patients ( $\mathrm{n}=7$ at baseline and $\mathrm{n}=12$ during follow-up) with PAWP values $>20 \mathrm{mmHg}$ during maximal exercise. Nevertheless, further analysis of our RHC data revealed that the increase in mPAP 
TABLE 2 Main haemodynamic and pulmonary function parameters and N-terminal pro-brain natriuretic peptide (NT-proBNP) at baseline and follow-up in patients using exercise echocardiography

\begin{tabular}{lccc} 
& Baseline & Follow-up & p-value \\
\hline sPAP50 mmHg & $38.0(30.0-43.8)$ & $43.0(36.0-50.3)$ & $<0.001$ \\
SPAP rest mmHg & $25.0(22.0-27.0)$ & $25.0(22.8-30.0)$ & 0.384 \\
sPAP peak mmHg & $43.2 \pm 11.7$ & $49.5 \pm 10.7$ & $<0.001$ \\
$V^{\prime} \mathbf{0}_{2}$ max \% pred & $79.5 \pm 23.6$ & $72.1 \pm 22.4$ & 0.001 \\
EqCo $_{2}$ L & $28.2 \pm 4.0$ & $30.2 \pm 3.9$ & $<0.001$ \\
FEV $\%$ pred & $97.8 \pm 15.8$ & $96.3 \pm 16.9$ & 0.148 \\
FVC \% pred & $102.8 \pm 17.2$ & $102.3 \pm 17.2$ & 0.691 \\
FEV 1 FVC \% & $80.9 \pm 6.1$ & $79.5 \pm 7.2$ & 0.009 \\
DLco cSB \% pred & $82.2(64.5-93.9)$ & $77.1(64.5-91.8)$ & 0.237 \\
DLco cVA \% pred & $83.7(71.8-94.9)$ & $82.4(69.6-90.3)$ & 0.045 \\
NT-proBNP pg·mL & $124.5 \pm 81.2$ & $155.3 \pm 117.2$ & 0.007
\end{tabular}

Data are presented as median (interquartile range) or mean \pm SD, unless otherwise stated. $n=58$. sPAP: systolic pulmonary arterial pressure; SPAP50: SPAP at $50 \mathrm{~W}$ exercise; $V^{\prime} \mathrm{O}_{2}$ max: maximal oxygen uptake; \% pred: \% predicted; $\mathrm{EqCO}_{2}$ : ventilatory equivalent for carbon dioxide at the ventilator threshold; $\mathrm{FEV} 1$ : forced expiratory volume in $1 \mathrm{~s}$; FVC: forced vital capacity; DLCO CSB: single breath diffusing capacity of lung for carbon monoxide corrected for haemoglobin; DLCO CVA: diffusing capacity of lung for carbon monoxide for alveolar volume corrected for haemoglobin.

during exercise between baseline and follow-up was not so much triggered by an increase in PAWP, but rather by an increase in the PAP/cardiac output slope and PVR (table 3 ). In addition, we did not observe a significant change in the PAWP/cardiac output slope between baseline and follow-up (table 3). These findings are in line with the hypothesis that the main driving force for progression of pulmonary hypertension in systemic sclerosis is progressive pulmonary vascular disease. Surprisingly, the increase in exercise PAP and PVR did not translate into a significant increase in the resting values (tables 2 and 3), suggesting that in early pulmonary vascular disease, exercise haemodynamics might be more sensitive to vascular remodelling than resting haemodynamics.

\section{Cardiopulmonary exercise test and NT-proBNP}

Peak oxygen uptake is acknowledged as a clinically relevant parameter in patients with pulmonary vascular disease $[1,37]$. In addition, it has been shown that decreased peak oxygen uptake may indicate PAH in patients with systemic sclerosis [38]. During follow-up, cardiopulmonary exercise testing revealed a significant increase in the ventilatory equivalent for carbon dioxide the ventilatory threshold, an indicator of pulmonary vascular disease. In addition, we found a mild but significant decrease in peak oxygen uptake over 4 years, which might be due to progression of pulmonary vascular disease, cardiac dysfunction or both.

FIGURE 2 Systolic pulmonary arterial pressure (sPAP) values at rest and at $50 \mathrm{~W}$. Comparison between baseline and follow-up after $3.9 \pm 0.9$ years in $\mathrm{n}=58$ patients undergoing exercise echocardiography.

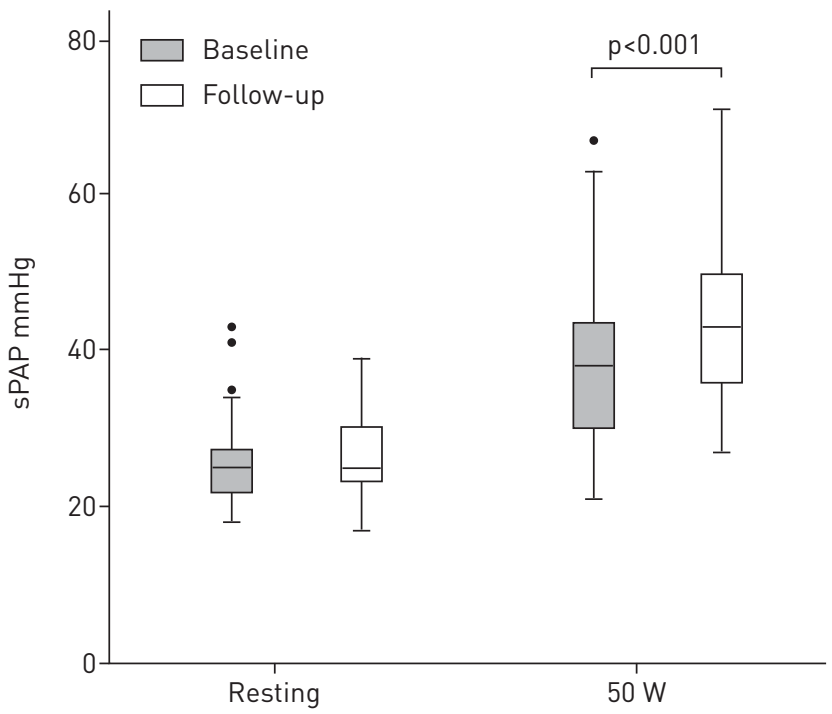


TABLE 3 Haemodynamic parameters derived from right heart catheterisation at baseline and follow-up

\begin{tabular}{|c|c|c|c|}
\hline & Baseline & Follow-up & p-value \\
\hline mPAP rest $\mathrm{mmHg}$ & $16.3 \pm 3.8$ & $16.8 \pm 3.6$ & 0.450 \\
\hline mPAP50 mmHg & $26.5(23.3-30.8)$ & $28.0(24.0-31.8)$ & 0.020 \\
\hline Heart rate rest beats $\cdot \min ^{-1}$ & $73.0 \pm 9.5$ & $69.8 \pm 8.7$ & 0.031 \\
\hline Heart rate $50 \mathrm{~W}$ beats $\mathrm{min}^{-1}$ & $111.4 \pm 12.4$ & $106.9 \pm 12.1$ & 0.016 \\
\hline mSAP rest $\mathrm{mmHg}$ & $87.0(83.0-94.0)$ & $82.0(75.0-93.0)$ & 0.009 \\
\hline mSAP50 mmHg & $104.5 \pm 12.4$ & $101.5 \pm 15.7$ & 0.240 \\
\hline PAWP rest $\mathrm{mmHg}$ & $7.6 \pm 3.1$ & $8.4 \pm 2.7$ & 0.154 \\
\hline PAWP $50 \mathrm{~W} \mathrm{mmHg}$ & $13.0 \pm 4.9$ & $14.6 \pm 5.8$ & 0.159 \\
\hline Cardiac output rest L.min ${ }^{-1}$ & $5.2 \pm 1.3$ & $4.9 \pm 1.3$ & 0.075 \\
\hline Cardiac output $50 \mathrm{~W} \mathrm{~L} \cdot \mathrm{min}^{-1}$ & $9.8 \pm 1.6$ & $9.0 \pm 1.4$ & 0.005 \\
\hline $\mathrm{RAP}$ rest $\mathrm{mmHg}$ & $4.6 \pm 2.6$ & $5.1 \pm 2.3$ & 0.285 \\
\hline RAP $50 \mathrm{~W}$ mmHg & $6.5(4.5-8.0)$ & $8.0(7.0-9.8)$ & 0.027 \\
\hline PVR rest dyn $\cdot \mathrm{s} \cdot \mathrm{cm}^{-5}$ & $141.6 \pm 62.3$ & $143.2 \pm 54.3$ & 0.801 \\
\hline PVR $50 \mathrm{~W}$ dyn.s. $\mathrm{cm}^{-5}$ & $120.7 \pm 55.6$ & $136.9 \pm 57.0$ & 0.002 \\
\hline $\mathrm{mPAP} / \mathrm{CO}$ slope $\# \mathrm{mmHg} \cdot \mathrm{L}^{-1} \cdot \mathrm{min}^{-1}$ & $2.4(1.7-3.6)$ & $2.6(2.2-3.7)$ & 0.049 \\
\hline PAWP/CO slope ${ }^{\#} \mathrm{mmH} \cdot \mathrm{L}^{-1} \cdot \mathrm{min}^{-1}$ & $1.13(0.40-1.89)$ & $1.16(0.65-2.14)$ & 0.471 \\
\hline
\end{tabular}

Data are presented as mean \pm SD or median (interquartile range), unless otherwise stated. $n=28 . m P A P$ : mean pulmonary arterial pressure; mPAP50: mPAP at $50 \mathrm{~W}$ exercise; mSAP: mean systemic arterial pressure; mSAP50: mSAP at $50 \mathrm{~W}$ exercise; PAWP: pulmonary arterial wedge pressure; RAP: right atrial pressure; PVR: pulmonary vascular resistance; CO: cardiac output. ${ }^{\#}$ : between rest and $50 \mathrm{~W}$.

We observed a mild but significant increase in NT-proBNP during follow-up. This may suggest increased cardiac stress. In addition, NT-proBNP is considered as a relevant prognostic factor for the development of PAH in systemic sclerosis [17, 39].

Mortality in the study period

Four out of 99 patients died during follow-up. The causes of death were malignant melanoma $(\mathrm{n}=1)$ and sepsis $(n=1)$, while in two cases the cause of death could not be determined. None of these patients showed any signs of PAH before death.

Role of medical therapy

$28 \%$ of our patients received bosentan for digital ulcers during the follow-up period, while this was only $7 \%$ at baseline. This may have ameliorated the haemodynamic results, as bosentan is a PAH drug and may

FIGURE 3 Increase in mean pulmonary arterial pressure ImPAP; cardiac output slope assessed between rest and $50 \mathrm{~W}$ ) at incremental cardiac output values during exercise. Comparison between baseline and follow-up right heart catheterisation in $n=28$ patients.

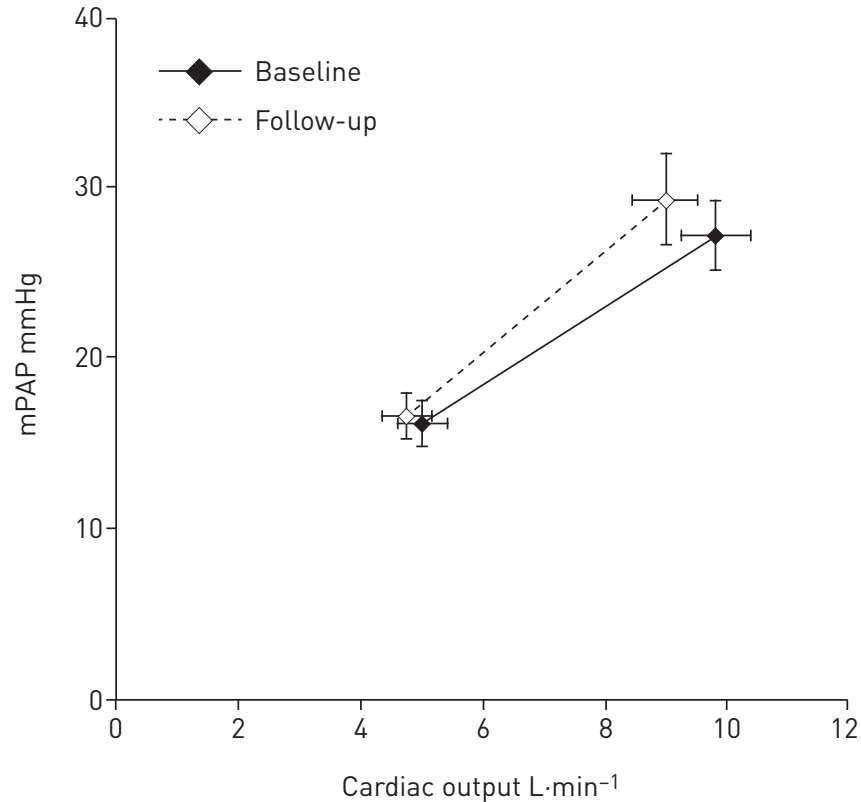


have prevented the development of PAH [40]. Nevertheless, in this subgroup of patients we found similar changes over time to those found in the rest of the cohort. Immunosuppressive therapy was applied in $29 \%$ of the patients at baseline and in $31 \%$ during follow-up, which also may have contributed to the clinical stability of patients [41]

The question remains as to whether the observed changes in pulmonary haemodynamics during exercise justify PAH therapy. Some pilot studies have been devoted to this question, with promising results [40, 42], but larger scale clinical trials will be needed to answer this important question.

\section{Limitations}

The relatively small number of patients included may be a limitation of our study. We excluded patients with significant left heart or lung disease, chronic thromboembolic pulmonary hypertension or PAH before enrolment. Therefore, we enriched the population with patients with a lower risk of development of pulmonary hypertension as compared with an average scleroderma cohort. Despite this, we observed a significant deterioration of pulmonary haemodynamics. This was primarily an echocardiography-based study; however, we performed RHC in a considerable portion of the patients and the results derived were in very good agreement with echocardiography. A further limitation may be the absence of a healthy control group. However, due to ethical reasons, invasive haemodynamic assessment is problematic in healthy subjects and currently there are no follow-up data available describing the changes in pulmonary exercise haemodynamics by noninvasive methods.

In conclusion, patients with systemic sclerosis developed mild deterioration of pulmonary exercise haemodynamics and exercise capacity over a 4-year follow-up period, indicating mild but significant progression of pulmonary vascular disease. The manifestation rate of $\mathrm{PAH}$ was $\geqslant 0.75$ cases per 100 patient-years.

\section{Acknowledgements}

We would like to thank Eugenia Lamont (Medical University of Graz, Graz, Austria) for careful linguistic review, as well as Daniela Kleinschek and Balazs Odler (Ludwig Boltzmann Institute for Lung Vascular Research, Graz) for their help in data management.

\section{References}

1 Galiè N, Humbert M, Vachiery JL, et al. 2015 ESC/ERS guidelines for the diagnosis and treatment of pulmonary hypertension: the Joint Task Force for the Diagnosis and Treatment of Pulmonary Hypertension of the European Society of Cardiology (ESC) and the European Respiratory Society (ERS). Eur Respir J 2015; 46: 903-975.

2 Hachulla E, Gressin V, Guillevin L, et al. Early detection of pulmonary arterial hypertension in systemic sclerosis: a French nationwide prospective multicenter study. Arthritis Rheum 2005; 52: 3792-3800.

3 Hachulla E, Carpentier P, Gressin V, et al. Risk factors for death and the 3-year survival of patients with systemic sclerosis: the French ItinérAIR-Sclérodermie study. Rheumatology 2009; 48: 304-308.

4 Khanna D, Gladue H, Channick R, et al. Recommendations for screening and detection of connective tissue disease-associated pulmonary arterial hypertension. Arthritis Rheum 2013; 65: 3194-3201.

5 Avouac J, Airò P, Meune C, et al. Prevalence of pulmonary hypertension in systemic sclerosis in European Caucasians and metaanalysis of 5 studies. J Rheumatol 2010; 37: 2290-2298.

6 Hachulla E, de Groote P, Gressin V, et al. The three-year incidence of pulmonary arterial hypertension associated with systemic sclerosis in a multicenter nationwide longitudinal study in France. Arthritis Rheum 2009; 60: 1831-1839.

7 Nihtyanova SI, Schreiber BE, Ong VH, et al. Prediction of pulmonary complications and long-term survival in systemic sclerosis. Arthritis Rheumatol 2014; 66: 1625-1635.

8 Codullo V, Caporali R, Cuomo G, et al. Stress Doppler echocardiography in systemic sclerosis: evidence for a role in the prediction of pulmonary hypertension. Arthritis Rheum 2013; 65: 2403-2411.

9 Meune C, Avouac J, Airò P, et al. Prediction of pulmonary hypertension related to systemic sclerosis by an index based on simple clinical observations. Arthritis Rheum 2011; 63: 2790-2796.

10 Reichenberger F, Voswinckel R, Schulz R, et al. Noninvasive detection of early pulmonary vascular dysfunction in scleroderma. Respir Med 2009; 103: 1713-1718.

11 Tyndall AJ, Bannert B, Vonk M, et al. Causes and risk factors for death in systemic sclerosis: a study from the EULAR Scleroderma Trials and Research (EUSTAR) database. Ann Rheum Dis 2010; 69: 1809-1815.

12 Chung L, Liu J, Parsons L, et al. Characterization of connective tissue disease-associated pulmonary arterial hypertension from REVEAL: identifying systemic sclerosis as a unique phenotype. Chest 2010; 138: 1383-1394.

13 Chung L, Farber HW, Benza R, et al. Unique predictors of mortality in patients with pulmonary arterial hypertension associated with systemic sclerosis in the REVEAL registry. Chest 2014; 146: 1494-1504.

14 Ruiz-Cano MJ, Escribano P, Alonso R, et al. Comparison of baseline characteristics and survival between patients with idiopathic and connective tissue disease-related pulmonary arterial hypertension. $J$ Heart Lung Transplant 2009; 28: 621-627.

15 Humbert M, Yaici A, de Groote P, et al. Screening for pulmonary arterial hypertension in patients with systemic sclerosis: clinical characteristics at diagnosis and long-term survival. Arthritis Rheum 2011; 63: 3522-3530.

16 Coghlan JG, Denton CP, Grünig E, et al. Evidence-based detection of pulmonary arterial hypertension in systemic sclerosis: the DETECT study. Ann Rheum Dis 2014; 73: 1340-1349. 
17 Allanore Y, Borderie D, Avouac J, et al. High N-terminal pro-brain natriuretic peptide levels and low diffusing capacity for carbon monoxide as independent predictors of the occurrence of precapillary pulmonary arterial hypertension in patients with systemic sclerosis. Arthritis Rheum 2008; 58: 284-291.

18 Hsu VM, Chung L, Hummers LK, et al. Development of pulmonary hypertension in a high-risk population with systemic sclerosis in the Pulmonary Hypertension Assessment and Recognition of Outcomes in Scleroderma (PHAROS) cohort study. Semin Arthritis Rheum 2014; 44: 55-62.

19 Kusunose K, Yamada H, Hotchi J, et al. Prediction of future overt pulmonary hypertension by 6-min walk stress echocardiography in patients with connective tissue disease. J Am Coll Cardiol 2015; 66: 376-384.

20 Kovacs G, Maier R, Aberer E, et al. Borderline pulmonary arterial pressure is associated with decreased exercise capacity in scleroderma. Am J Respir Crit Care Med 2009; 180: 881-886.

21 Condliffe R, Kiely DG, Peacock AJ, et al. Connective tissue disease-associated pulmonary arterial hypertension in the modern treatment era. Am J Respir Crit Care Med 2009; 179: 151-157.

22 Kovacs G, Maier R, Aberer E, et al. Assessment of pulmonary arterial pressure during exercise in collagen vascular disease: echocardiography $v s$ right-sided heart catheterization. Chest 2010; 138: 270-278.

23 Kovacs G, Avian A, Pienn M, et al. Reading pulmonary vascular pressure tracings. How to handle the problems of zero leveling and respiratory swings. Am J Respir Crit Care Med 2014; 190: 252-257.

24 Naeije R, Vanderpool R, Dhakal BP, et al. Exercise-induced pulmonary hypertension: physiological basis and methodological concerns. Am J Respir Crit Care Med 2013; 187: 576-583.

25 Herve P, Lau EM, Sitbon O, et al. Criteria for diagnosis of exercise pulmonary hypertension. Eur Respir J 2015; 46 : 728-737.

26 Callejas-Rubio JL, Moreno-Escobar E, de la Fuente PM, et al. Prevalence of exercise pulmonary arterial hypertension in scleroderma. J Rheumatol 2008; 35: 1812-1816.

27 Steen V, Chou M, Shanmugam V, et al. Exercise-induced pulmonary arterial hypertension in patients with systemic sclerosis. Chest 2008; 134: 146-151.

28 Alkotob ML, Soltani P, Sheatt MA, et al. Reduced exercise capacity and stress-induced pulmonary hypertension in patients with scleroderma. Chest 2006; 130: 176-181.

29 Collins N, Bastian B, Quiqueree L, et al. Abnormal pulmonary vascular responses in patients registered with a systemic autoimmunity database: Pulmonary Hypertension Assessment and Screening Evaluation using stress echocardiography (PHASE-I). Eur J Echocardiogr 2006; 7: 439-446.

30 D'Alto $\mathrm{M}$, Ghio S, D'Andrea $\mathrm{A}$, et al. Inappropriate exercise-induced increase in pulmonary artery pressure in patients with systemic sclerosis. Heart 2011; 97: 112-117.

31 Kovacs G, Avian A, Tscherner M, et al. Characterization of patients with borderline pulmonary arterial pressure. Chest 2014; 146: 1486-1493.

32 Fox BD, Shimony A, Langleben D, et al. High prevalence of occult left heart disease in scleroderma-pulmonary hypertension. Eur Respir J 2013; 42: 1083-1091.

33 Baptista R, Serra S, Martins R, et al. Exercise-induced pulmonary hypertension in scleroderma patients: a common finding but with elusive pathophysiology. Echocardiography 2013; 30: 378-384.

34 Voilliot D, Magne J, Dulgheru R, et al. Determinants of exercise-induced pulmonary arterial hypertension in systemic sclerosis. Int J Cardiol 2014; 173: 373-379.

35 Gargani L, Pignone A, Agoston G, et al. Clinical and echocardiographic correlations of exercise-induced pulmonary hypertension in systemic sclerosis: a multicenter study. Am Heart J 2013; 165: 200-207.

36 Saggar R, Khanna D, Furst DE, et al. Exercise-induced pulmonary hypertension associated with systemic sclerosis: four distinct entities. Arthritis Rheum 2010; 62: 3741-3750.

37 Wensel R, Francis DP, Meyer FJ, et al. Incremental prognostic value of cardiopulmonary exercise testing and resting haemodynamics in pulmonary arterial hypertension. Int J Cardiol 2013; 167: 1193-1198.

38 Dumitrescu D, Nagel C, Kovacs G, et al. Cardiopulmonary exercise testing for detecting pulmonary arterial hypertension in systemic sclerosis. Heart 2017; 103: 774-782.

39 Avouac J, Meune C, Chenevier-Gobeaux C, et al. Cardiac biomarkers in systemic sclerosis: contribution of high-sensitivity cardiac troponin in addition to N-terminal pro-brain natriuretic peptide. Arthritis Care Res 2015; 67: $1022-1030$

40 Kovacs G, Maier R, Aberer E, et al. Pulmonary arterial hypertension therapy may be safe and effective in patients with systemic sclerosis and borderline pulmonary artery pressure. Arthritis Rheum 2012; 64: 1257-1262.

41 Miyamichi-Yamamoto S, Fukumoto $\mathrm{Y}$, Sugimura $\mathrm{K}$, et al. Intensive immunosuppressive therapy improves pulmonary hemodynamics and long-term prognosis in patients with pulmonary arterial hypertension associated with connective tissue disease. Circ J 2011; 75: 2668-2674.

42 Saggar R, Khanna D, Shapiro S, et al. Brief report: effect of ambrisentan treatment on exercise-induced pulmonary hypertension in systemic sclerosis: a prospective single-center, open-label pilot study. Arthritis Rheum 2012; 64: $4072-4077$. 